\title{
La(s) industria(s) de la migración. ¿Un nuevo enfoque en el análisis de la movilidad internacional?
}

The migration industries. A new perspective in the analysis of
international migration?

\author{
Ana López-SALA \\ Consejo Superior de Investigaciones Científicas \\ ana.Isala@cchs.csic.es (ESPAÑA)
}

Recibido: 25.102018

Aceptado: 30.01 .2020

\section{RESUMEN}

A lo largo de la última década el enfoque de la industria migratoria ha tenido un amplio desarrollo en los estudios sobre la movilidad internacional de personas. Esta perspectiva ha tenido un particular desarrollo en la literatura de intersección sobre las nuevas formas que ha adoptado la regulación migratoria y los mecanismos de intermediación y privatización. Se trata de un enfoque en construcción donde todavía permanecen abiertas amplias controversias en torno a su contorno, alcance, actores y funciones. Sin embargo, esta literatura ha realizado importantes contribuciones a los estudios sobre la movilidad internacional de trabajadores - a través fundamentalmente del análisis de la intermediación laboral- y la gestión del control migratorio -a través de la conformación de alianzas de nuevo cuño entre el Estado y diversos actores privados. El objetivo de este artículo es presentar y analizar la conformación de este enfoque, los dilemas que han emergido en el seno de esta literatura, así como reflexionar críticamente en torno a sus principales contribuciones al campo de los estudios migratorios. En este sentido, y enmarcado en el presente monográfico, el artículo pretende realizar una aportación que transcienda la mera descripción de esta perspectiva para interrogarse, entre otros, sobre sus peculiaridades como enfoque, su diálogo con otras orientaciones influyentes en los estudios migratorios, o, de forma muy

${ }^{1}$ Este artículo ha sido elaborado en el marco del proyecto MIND ( $«$ Actores no estatales del control migratorio: Una aproximación desde la Migration Industry»). Plan Nacional (Excelencia). Referencia: CSO2014-53680. 
sucinta, sus vínculos con otras teorías. Este recorrido pretende servir también para introducir esta perspectiva en la literatura española, ya que, a pesar del interés que ha despertado a nivel internacional, apenas se ha incorporado a la investigación sobre las migraciones en España.

\title{
PALABRAS CLAVE
}

Industria de la migración, privatización, intermediación, control migratorio, mercado laboral.

\begin{abstract}
Throughout the past decade the migration industry approach has been broadly used in studies on the international mobility of people. In particular, it has been the focus of literature examining how new forms of migration regulation have converged with intermediation and privatization mechanisms. This approach is still being developed and there continue to be wide-ranging debates regarding its contours, scope, actors and functions. However, this literature has provided important contributions to the study of the international mobility of workers, mainly through analyses of the novel partnerships that have been established between the state and various private actors to provide labor intermediation and migration control management. The objective of this article is to present and analyze the composition of this focus and the dilemmas that have emerged within the literature, as well as to provide a critical reflection regarding its main contributions to the field of migration studies. In this sense, framed within this monograph, the article seeks to go beyond providing a mere description of the perspective to examine, among other aspects, its peculiarities as a focus and its discourse with other influential approaches in migration studies or, succinctly put, its links with other theories. It will also serve to introduce this perspective to the Spanish literature, as, despite the interest it has sparked on an international level, it has barely been incorporated into research on migrations in Spain.
\end{abstract}

\section{KEY WORDS}

Migration Industry, Privatization, Intermediation, Migration Control, Labour Markets. 


\section{INTRODUCCIÓN}

A lo largo de la última década el concepto de industria migratoria ha ganado protagonismo en el léxico académico de los estudios sobre la movilidad internacional. Aunque sus orígenes pueden rastrearse varias décadas atrás, el reciente «auge» de este enfoque se enmarca en la incorporación analítica de los actores privados en el estudio de los determinantes y la modulación de las migraciones. El alejamiento del determinismo estatal que ha imperado en los estudios sobre la movilidad humana y su concreción a través del enfoque de la industria de la migración ha ofrecido nuevas perspectivas para entender la articulación contemporánea de las interacciones entre los Estados, el sector privado y los propios migrantes. Adicionalmente, su desarrollo como prisma analítico parte de la consideración de que las dinámicas y la gestión de los flujos migratorios internacionales no pueden entenderse adecuadamente sin tomar en consideración tanto los procesos de mercantilización y generación de beneficios que cristalizan en torno a ellos, como las prácticas neoliberales que han alcanzado a muchas de las esferas de la gestión migratoria. Es por tanto en la literatura de intersección sobre las nuevas formas que ha adoptado la regulación migratoria y los mecanismos de intermediación y privatización donde este enfoque ha recibido mayor atención.

Se trata, sin embargo, de una perspectiva todavía en vías de consolidación, en donde siguen abiertas amplias controversias, no sólo en torno a la propia definición del concepto, sino, como veremos, en torno a su alcance, contorno, actores y antecedentes. Muchos de los contenidos, consensos y disensos que han aparecido en estos debates son analizados en este artículo, cuyo objetivo es presentar y analizar la conformación de este enfoque, los dilemas que han emergido en el seno de esta literatura, así como reflexionar críticamente en torno a sus principales contribuciones al campo de los estudios migratorios. En este sentido, y enmarcado en el presente monográfico, el artículo pretende realizar una aportación que transcienda la mera descripción de esta perspectiva para interrogarse, entre otros, sobre sus peculiaridades como enfoque, su diálogo con otras orientaciones influyentes en los estudios migratorios, o, de forma muy sucinta, sus vínculos con otras teorías. Este recorrido pretende servir también para introducir esta perspectiva en la literatura española, ya que, a pesar del interés que ha despertado esta perspectiva a nivel internacional, apenas se ha incorporado a la investigación sobre las migraciones en España².

El artículo se divide en dos partes principales. En la primera se transita desde la conformación del concepto a la cimentación del enfoque, presentándose distintas visiones y revisiones sobre ellos a lo largo de estas dos últimas décadas. En esta primera parte se subrayan también los elementos que construyen el tronco común de estas aportaciones, así como sus elementos más distintivos y

2 Algunas excepciones son los estudios de López-Sala y Godenau (2016, 2017). Sin embargo, si pensamos en sus antecedentes es posible incorporar en esta literatura los estudios que se han detenido en la "intermediación laboral" en los enclaves españoles de agricultura intensiva (véase, por ejemplo Pedreño, 2014). 
significativos. La segunda parte del artículo se detiene en aquellas esferas donde esta perspectiva ha tenido mayor desarrollo y alcance, recorriéndose las principales contribuciones de los estudios en torno a las industrias migratorias, los avances que han propuesto y los retos y reflexiones que han suscitado. Se trata, en conjunto, de un artículo que discurre por el estado actual de la investigación sobre las industrias migratorias y que se interroga sobre su alcance teórico y sus potencialidades contribuciones para densificar los análisis y la comprensión de la migración internacional.

\section{DE LA «FABRICACIÓN» DEL CONCEPTO AL ENFOQUE DE LA INDUSTRIA MIGRATORIA}

A pesar de su popularidad actual, es posible encontrar amplios antecedentes de este enfoque ${ }^{3}$, aunque no siempre haya adoptado esta denominación, en la literatura de los estudios migratorios. A finales de los años setenta Harney introdujo el concepto de «comercio de la inmigración» para referirse a las actividades lucrativas desarrolladas por un amplio abanico de intermediarios -principalmente brokers laborales y de transporte, así como prestamistas- que intervinieron en la facilitación y el mantenimiento de la migración italiana hacia el continente americano entre finales del siglo XIX y principios del XX (Harney, 1977). A esta mención inicial le sucede en las décadas siguientes otras aportaciones que emplean términos como «negocio de la inmigración», para describir los procesos que impulsan el tráfico de migrantes, (Salt y Stein, 1997; Kyle y Koslowski, 2001), o «mercaderes del trabajo ${ }^{4}$, para referirse a los agentes privados que movilizan trabajadores a través de las fronteras con el objetivo de cubrir las demandas laborales de los países de destino (Martin, 2005). Estas aportaciones «pioneras» subrayaban la orientación basada en la búsqueda de beneficios que genera y estructura esta industria y su encaje e influencia en la dinámica de los flujos internacionales de población.

En su denominación actual, el término «industria de la migración» aparece por primera vez en 1997 en Global Diasporas, donde Robin Cohen la define como «el conjunto de agentes de viaje, abogados, reclutadores e intermediarios que sustentan los vínculos migratorios entre países de origen y de destino» (Cohen, 1997) y, unos años más tarde en The Age of Migration, donde Stephen Castles y Mark J. Miller, emplean el concepto vinculándolo a la teoría de los sistemas migratorios (Castles and Miller, 2003; Castles, 2007). A diferencia de las

\footnotetext{
3 Algunos autores han subrayado la longevidad del enfoque (Light, 2013; Hernández-León, 2012), más allá de su denominación actual. Así se observa en los estudios históricos que abordaron el papel seminal de diversos actores intermediarios en el impulso y el mantenimiento de la emigración europea hacia el continente americano (veáse Jenks y Lauck, 1922; Fairchild, 1925; y más recientemente Gabaccia, 2000).

${ }^{4}$ El concepto de «mercaderes del trabajo» puede rastrearse asimismo varias décadas atrás en diversas publicaciones sobre el Programa Bracero (véase Galarza, 1964; y más recientemente Calavita, 1992).
} 
formulaciones previas, estas definiciones analizaron la industria en el contexto de dinámicas migratorias en curso y subrayaron su surgimiento como respuesta a las necesidades de servicios que demandaban los migrantes a lo largo de todo el proceso migratorio. En estas aportaciones la industria migratoria se concibe como uno de los factores explicativos en el mantenimiento de los flujos en un determinado contexto migratorio.

Pero sin duda el término cobra impulso a mediados de la década pasada a través de los trabajos de Hernández-León, cuando en su estudio sobre la migración entre México y Estados Unidos, define la industria de la migración como «el conjunto de servicios, inversores y empresarios que, motivados por la obtención de beneficios, facilitan y sostienen la migración internacional» (Hernández-León, 2005). Su definición incluye distintas actividades (formales e informales; legales e ilegales) y actores, que interactúan con los Estados y los migrantes, estructurando el proceso migratorio (Hernández-León, 2005). En esta aproximación el beneficio económico se articula como un factor que promueve y mantiene los flujos migratorios transnacionales más allá de las políticas y las constricciones estatales. En los años finales de la década pasada, al hilo de su investigación sobre los flujos a través de la frontera mexicano-estadounidense, David Spener se preguntaba si la industria migratoria podía considerarse un concepto analítico plenamente desarrollado, o más bien, una útil figura retórica para referirse al conjunto de actores -y acciones- dedicados a la facilitación de la migración irregular (Spener, 2010).

En fechas recientes el término ha sido objeto de nuevas revisiones que muestran su carácter como un enfoque todavía en construcción. La discusión se ha centrado tanto en el contenido y modulación del propio concepto - con alternativas quizás más amplias y complejas que focalizan su análisis, de forma genérica, en el proceso de intermediación («infraestructura de la migración»; Xiang y Lindquist, 2014), o que se han centrado en las funciones, actores y áreas donde se produce esta intermediación («mercados migratorios»; Nyberg-Sorensen y Gammeltoft-Hansen, 2012; Nyberg-Sorensen, 2012; López-Sala y Godenau, 2016 y 2017)- como en su alcance. Es esta última dimensión la que ha acaparado una mayor atención en los últimos años, a través de esfuerzos analíticos que han pretendido, de forma más exhaustiva, describir las múltiples funciones de la industria migratoria, mapear los actores que intervienen (generalmente motivados por la generación de beneficios) y explorar sus efectos tanto sobre los patrones migratorios, como sobre la gestión de la movilidad internacional. En concreto, los estudios sobre la industria migratoria han ampliado su alcance a través de una visión más comprehensiva que se detiene no sólo en la facilitación de la migración, sino en la restricción y control de los flujos, así como en las labores de asistencia y acogida a los migrantes. Resulta en este sentido paradigmático, por su carácter estructurante y su influencia en los estudios posteriores, el trabajo compilatorio de Gammeltoft-Hansen y Nyberg Sorensen en donde se define la industria migratoria como «la variedad de actores no estatales que suministran servicios que facilitan, restringen o asisten a la migración internacional» (Gammeltoft-Hansen y Nyberg-Sorensen, 2013). Esta clasificación por funciones, 
y sus actores asociados, que ha llevado también a algunos autores a hablar recientemente de las industrias de la migración (Cranston, Schapendonk y Spaan, 2018), ha enriquecido este enfoque con investigaciones centradas en el ámbito de la restricción migratoria (Lemberg-Pedersen, 2013), así como con estudios comparados desde el denominado «principal-agent framework» (Surak, 2018).

Todas estas contribuciones han ido conformando un corpus de literatura académica con un creciente protagonismo en los estudios migratorios que ha mostrado recientemente ciertos signos de consolidación. Se trata sin embargo todavía de un campo emergente sometido a continuo escrutinio a través de nuevas reflexiones, ampliaciones teóricas y estudios de caso. ¿Cuáles son los elementos comunes que encontramos en estas aportaciones? ¿Qué aspectos pueden ser señalados como los más significativos y específicos de este enfoque? ¿Qué acuerdos, desacuerdos y discusiones aparecen en la literatura actual sobre las industrias migratorias? ¿En qué esferas de los estudios migratorios ha tenido un mayor desarrollo?

Un punto de partida común en este enfoque ha sido la introducción de los beneficios y de la rentabilidad económica como lógicas que activan la intermediación en la movilidad internacional ${ }^{5}$. Los estudios sobre las industrias migratorias han mostrado la influencia de la oportunidad de ganancia y la búsqueda de lucro, materializada en gran parte a través de actividades y servicios suministrados por distintos tipos de actores, en la modulación de las dinámicas y los patrones migratorios. También se ha detenido en profundizar en el mantenimiento, en el sostenimiento en el tiempo y en el espacio de los flujos más allá de coyunturas políticas y económicas. De ahí las reflexiones que ha ofrecido en torno a sus vínculos -e interrelaciones- con las redes sociales y su capacidad estructurante ${ }^{6}$

${ }^{5}$ La industria migratoria, han sostenido diversos autores, puede entenderse como un marco analítico propio, como unas nuevas lentes a través de las cuáles estudiar los flujos internacionales de población (Hernández-León, 2013; Gammeltoft-Hansen y Nyberg Sorensen, 2013). Esta posición, sin embargo, no ha evitado que se señalen también sus debilidades, en especial, que se trata de una perspectiva todavía demasiado descriptiva que muestra por el momento cierta debilidad teórica; una debilidad que debe fortalecerse a través de análisis más específicos que sirvan para entender tanto la relación entre distintos actores privados en la organización social de la migración (Hernández-León, 2013), como el funcionamiento y la intersección de esta industria con la propia movilidad internacional (Cranston, Schapendonk y Spaan, 2018).

${ }^{6}$ Durante las tres últimas décadas los estudios migratorios han subrayado el papel de las redes sociales, basadas en relaciones de parentesco, co-etnicidad y/o comunidad, para fomentar y mantener los flujos migratorios. Sin embargo, como subrayó Krissman a mediados de la década pasada la teoría de redes no tiene la solidez explicativa que se le ha atribuido al obviar el impacto en los procesos migratorios de un amplio abanico de actores e instituciones ajenas a las redes personales (Krissman, 2005). Esta crítica ha suscitado un amplio debate analítico en torno a la complementariedad/suplementariedad de las redes personales y las redes «comercializadas». En algunos contextos, sin embargo, los límites entre estos dos tipos de redes parecen haberse tornado crecientemente difusos. Así, por ejemplo, los estudios de la industria migratoria han señalado: a) tanto el efecto de las políticas fronterizas en la paulatina mercantilización del apoyo previamente provisto por las redes personales o comunitarias, como, b) el carácter relativamente altruista de esta intermediación en el caso de algunos actores que han sido etiquetados tradicionalmente como actores movidos, en exclusiva, por la obtención de beneficios (véase Spener, 2011; Nyberg-Soren- 
(Krissman, 2005; Hernández-León, 2012; Lindquist, Xiang y Yeoh, 2012; Zabko, Aasland y Birgit-Endresen, 2018). Por todo ello, la industria de la migración ha sido considerada una mesoestructura, que conecta la macroestructura con las microestructuras de la migración ${ }^{7}$ (Spener, 2010) incorporándose a las aproximaciones de rango medio que se centran en el mantenimiento de los flujos ${ }^{8}$.

Como veremos, el énfasis en el examen de la función de intermediación ha ofrecido también nuevas perspectivas para entender la urdimbre de las interacciones contemporáneas entre el sector privado, los Estados, las organizaciones sociales y los movimientos de personas. Se ha destacado, por ejemplo, la influencia de los actores privados en la conformación de las migraciones, aportando una perspectiva que transciende la mera, y a veces simplista, descripción de la privatización de su gestión ${ }^{9}$. Por otra, los estudios sobre la industria migratoria se han desplazado más allá de las lógicas conceptuales y metodológicas del Estado nacional investigando las redes comerciales y las instituciones informales especializadas en trascender fronteras (Cranston, Schapendonk y Spaan, 2018) y subrayando el intenso carácter transnacional de la facilitación y del control migratorio (Nyberg Sorensen, 2012).

Esta literatura comparte además la consideración de que esta industria ha llegado a reestructurar la gobernanza global de la movilidad humana, tanto a nivel nacional como internacional. Como sostiene Betts, la industria migratoria ha llegado a constituirse como una faceta crecientemente influyente en esta gobernanza, a pesar de que su papel ha sido generalmente obviado bajo la asunción de que la migración es, primordialmente, "una modalidad estatista de gobernanza" (Betts, 2013, 49).

Durante los últimos años el enfoque de la industria migratoria ha entrado también en diálogo con el denominado «mobility turn» (Cresswell, 2006; Sheller y Urry, 2006), una perspectiva que se detiene en entender qué es lo que sucede «on the move» ${ }^{10}$ y que reconceptualiza la migración como un proceso en curso,

sen, 2013; Stone-Cadena y Álvarez, 2018). Otros autores han subrayado el carácter dinámico de las redes sociales en los procesos de control/facilitación migratoria (Schapendonk, 2018).

${ }^{7}$ Esta postura ha sido frecuente en gran parte de los estudios que adoptan este enfoque y que la posicionan como una meso-estructura intermedia entre las redes sociales y el Estado y las instituciones internacionales.

${ }^{8}$ Más recientemente, a través de los resultados que han ofrecido algunos estudios de caso, se ha sostenido que la industria migratoria no sólo interviene en el mantenimiento de los flujos, sino que se trata de un factor que explica la activación de los flujos, su dinámica, composición y dirección a través, entre otros, de sus efectos sobre las decisiones migratorias (véase Spaan y van Naerssen, 2018).

9 Investigaciones desarrolladas en el seno de este enfoque (Bloom, 2016; Bacon, 2005) han subrayado que una parte importante de la gestión migratoria, tradicionalmente asociada a la condición soberana del Estado, ha sido delegada, externalizada, a actores no públicos a través, entre otros, de procesos de licitación competitiva o de la firma de acuerdos y convenios. Estos acuerdos público-privados han tomado forma en áreas de intervención pública muy variadas como la regulación de los flujos migratorios laborales, la vigilancia fronteriza o los dispositivos de internamiento (véase López-Sala y Godenau, 2017).

${ }^{10}$ Este enfoque, muy influyente en las ciencias sociales durante la última década, cuestiona las visiones «sedentaristas» de lo social y considera la migración como un proceso que «se produ- 
más que como un evento (Schapendonk y Steel, 2014). El «mobility turn» subraya la diversidad de formas que han adoptado los movimientos transfronterizos, transcendiendo y poniendo en entredicho la visión más ortodoxa de la migración, en referencia al paradigma de movilidad laboral de largo plazo. Explicar estas formas mucho más complejas de movilidad exige incorporar analíticamente otro tipo de actores, ampliando su alcance y caracterización. En segundo lugar, como han sostenido Cranston, Schapendonk y Spaan, el paradigma del «mobility turn» se ha detenido en explicar cómo los procesos de movilidad de los migrantes se ven afectados por formas de cooperación y de colisión entre los intereses y las estrategias de distintos actores que simultáneamente y dinámicamente modulan el proceso (Cranston, Schapendonk y Spaain, 2018; Schapendonk, 2018).

Durante la década pasada la investigación más sobresaliente en torno a la industria migratoria se centró en los actores privados ilícitos implicados en la facilitación de la migración irregular, en su capacidad para circunvalar las fronteras estatales y sostener los flujos a pesar de las políticas restrictivas (Salt and Stein, 1997; Kyle and Koslowski, 2001; Hérnandez-León, 2005; Spener, 2009). Sin embargo, más recientemente, el enfoque de la industria migratoria ha destacado tanto en el análisis de la intermediación laboral, como en los estudios sobre la restricción migratoria; a los que se han unido más recientemente los que se detienen en la denominada industria de la asistencia o del rescate. ¿Cómo han discurrido los análisis en cada una de estas esferas? ¿Cuáles han sido sus aportaciones más importantes?¿Qué aspectos han despertado mayor atención? ¿Cómo se han encajado las aportaciones de estas piezas de la literatura en otras aproximaciones de los estudios migratorios?

\section{LA INDUSTRIA MIGRATORIA Y LA MOVILIDAD INTERNACIONAL DE TRABAJADORES: LA CENTRALIDAD DEL ANÁLISIS SOBRE LA INTERMEDIACIÓN LABORAL.}

El estudio de la intermediación como elemento determinante en la canalización de los flujos laborales de trabajadores migrantes ha sido particularmente prolífico. La historiografía sobre la gran emigración europea hacia Norteamérica ya dio cuenta del papel crucial de los agentes laborales en la promoción de estos flujos a través de figuras como los «enganchadores» o los «padroni» (Harney, 1977; Gabaccia, 2000). En las últimas décadas del siglo pasado la intermediación laboral vuelve a cobrar protagonismo explicativo en el análisis de los flujos migratorios de mexicanos hacia Estados Unidos (Massey, Alarcón, Durand y González, 1987; Krissman, 2000). En esta literatura sobre algunos de los sistemas migratorios más conocidos, la intermediación laboral se concibe como un proceso al servicio de la demanda del mercado de trabajo de las economías

ce» en movimiento. Su principal objetivo es entender cómo las movilidades son producidas, constreñidas y contestadas. Este enfoque ha tenido además una importante influencia en los estudios sobre negociación identitaria en contextos migratorios (véase Fernández-Montes, 2013). 
receptoras que facilita, canaliza y distribuye la fuerza de trabajo migrante de acuerdo con las fuerzas del mercado, contrarrestando, ofreciendo un contrapeso frente a los procesos restrictivos que producen los regímenes fronterizos. Más recientemente y ya bajo la etiqueta de «industria de la migración», esta mediación laboral destaca en el análisis de los flujos laborales temporales y estacionales de baja cualificación, en donde los factores de la estructura del mercado de trabajo de los países receptores se conjuga con el ensayo de nuevas fórmulas de regulación (Hennebry, 2008; Surak, 2018; Hernández-León, 2017) incrustando el estudio de la intermediación en la constelación de las potenciales interacciones entre el Estado, los migrantes, las agencias laborales y los intermediarios informales. Esta parte de la literatura se ha detenido no sólo en analizar el efecto de la implantación de estos sistemas de regulación en el surgimiento de esta industria, alimentada por las posibilidades de negocio que genera la movilización de trabajadores temporales, sino en sus efectos sobre la producción de una fuerza de trabajo inmigrante crecientemente vulnerable y sometida a procesos de inseguridad y flexibilidad ${ }^{11}$ (Kushnirovich, Raijman y Barak-Bianco, 2018; Consterdine y Samuk, 2018). Los efectos de la industria migratoria y su funcionamiento en la estructuración de los mercados laborales también ha despertado el interés académico, fundamentalmente en el caso de Europa, donde algunos estudios más recientes se han interrogado sobre las interconexión entre distintos actores, entre otros, agencias estatales, empresarios y agencias de reclutamiento, en la estructuración del sistema migratorio, la conformación de mercados de trabajo étnicamente segmentados y la incorporación laboral de los extranjeros (Garapich, 2008; Findlay y McCollum, 2013).

El análisis del rol que ejercen los intermediarios laborales ha sido entendido también como una vía para abrir, para entender, la denominada «caja negra» de los estudios migratorios (Lindquist, Xiang y Yeoh, 2012). Como sostienen estos autores, aunque la investigación sobre las migraciones se ha ocupado profusamente de explicar las causas que llevan a los migrantes a abandonar sus lugares de origen y lo que sucede tras su llegada al destino, poco se conoce sobre las formas de infraestructura que condicionan su movilidad, en concreto, cómo se posibilita la movilidad y cómo es organizada por los brokers, particularmente en el proceso de reclutamiento. Los estudios sobre la intermediación laboral han sido particularmente abundantes en el análisis de los regímenes contemporáneos de migración trasnacional en el sistema migratorio Asia-Pacífico (Xiang, 2012; Kern y Müller-Böker, 2015; Franck, Arellano y Anderson, 2018; Goh, Wee y Yeoh, 2017). En estos se ha profundizado en las dinámicas interdependientes entre el Estado y la industria migratoria en la gobernanza de la movilidad, sus efectos sobre las decisiones migratorias (Spaan y van Naerssen, 2018), problematizándose, adicionalmente, a través de un buen número de estudios de caso,

11 También han sido destacados los efectos de esta industria en las prácticas de tráfico de trabajadores y en formas de explotación laboral y de trabajo forzoso. Véase, por ejemplo, para el caso de Israel los trabajos de Kemp y Raijam (2014). 
la tradicional «demonización» de los intermediarios laborales (McKeown, 2012; Baas, 2018).

El análisis de estos intermediarios laborales se ha incorporado también, hace apenas unos años, a la investigación sobre los flujos de migrantes altamente cualificados, un segmento tradicionalmente obviado en la literatura sobre la industria migratoria. En 2015, Groutsis, van den Broek y Harvey destacaban que esta intermediación podía no sólo tener un papel fundamental en la adopción de las decisiones migratorias, sino que a través del suministro de información y de servicios podía mejorar la capacidad de elección de los migrantes y contener los riesgos que asumían en el proceso (Groutsis, van den Broek y Harvey, 2015); pero también segmentar, según categorías, tanto su acceso al mercado de trabajo, como su éxito laboral en los países de destino (van den Broek, Harvey y Groutsis, 2016). La todavía escasa literatura sobre la intermediación en este tipo de movilidad, mucha de ella centrada en los flujos intraeuropeos, ha subrayado otros dos aspectos: a) en primer lugar, que la industria migratoria y las redes sociales no pueden ser consideradas subsidiarias, sino que se encuentran ampliamente interconectadas y operan de forma simultánea; $y, b$ ) en segundo lugar, en los efectos de esta industria en la selección de los destinos migratorios y en la canalización y conformación del sistema migratorio europeo.

A pesar de que, como se ha mencionado previamente en este artículo, nos encontramos todavía ante un enfoque de investigación emergente y en construcción, donde las aportaciones son todavía muy fragmentadas, puede indicarse que la investigación sobre las industrias migratorias desde la perspectiva del mercado de trabajo ha realizado hasta la fecha algunas contribuciones dignas de señalar. De forma muy sucinta, los análisis sobre la intermediación laboral han ido más allá de enclavar su papel en la conformación de los patrones migratorios para detenerse en sus efectos estructurantes sobre el mercado de trabajo y sobre la creciente precariedad laboral de los migrantes. En segundo lugar, han dotado de mayor complejidad a los modelos explicativos de la adopción de las decisiones migratorias a través de estudios de caso que profundizan en el papel de esta industria migratoria no sólo en la decisión de migrar per se, sino en el tipo de proyecto migratorio, o incluso, en las decisiones sobre la elección del destino. En tercer lugar, han subrayado su papel a la hora de reproducir trasnacionalmente podríamos decir, los privilegios de cierto tipo de migrantes. Esta industria, indican, por tanto, interviene no sólo en la producción, sino en la reproducción de categorías de migrantes. Por último, han ayudado a prestar más atención a los vínculos entre distintos actores en la gestión de la movilidad laboral de trabajadores, incorporando una perspectiva más amplia que no sólo se detiene en los intermediarios, sino que profundiza en sus vínculos con empresarios, migrantes y agencias estatales subrayando los acuerdos, alianzas y tensiones que se producen entre actores en esta esfera. 


\section{LA INDUSTRIA DEL CONTROL MIGRATORIO: LA INCORPORACIÓN DE LOS ACTORES PRIVADOS EN LA RESTRICCIÓN DE LA MOVILIDAD.}

En la última década los análisis desde el enfoque de la industria migratoria han cobrado también impulso en el estudio y la indagación sobre el control migratorio. Se trata de una parte de la literatura mucho más fragmentada que incorpora un amplio abanico de actores y esferas de gestión. A pesar de ello comparte un punto de partida común y es el de la constatación de la creciente participación de actores no estatales en la implantación de las medidas de restricción migratoria. Son varios los términos que han empleado los especialistas, desde la aportación pionera de Lahav (Lahav, 1998, 2000) para etiquetar este nuevo rasgo, entre otros, la «privatización», el «outsourcing» o la «delegación» del control migratorio (Bloom, 2015; Andrijasevic, 2015; López-Sala y Godenau, 2017). La introducción en el análisis de estos nuevos actores privados, desde un prisma que subraya las funciones de intermediación, supone un cambio importante en la orientación más ortodoxa del estudio del control migratorio, que ha adolecido de un excesivo determinismo estatal, sólo contrarrestado recientemente por los enfoques que han subrayado las formas de acción, agencia y resistencia de los propios migrantes frente a la conformación de los regímenes fronterizos.

Esta literatura ha llevado a primera línea de análisis, el creciente protagonismo de estos actores privados en la gobernanza nacional y global de la movilidad (Betts, 2013). Además, a través del examen de distintas categorías de actores, ha dado buena cuenta de la multiplicidad de fórmulas que esta colaboración pública-privada ha adoptado, desde la externalización completa a la delegación parcial (López-Sala y Godenau, 2017; López-Sala y Godenau, 2019). En tercer lugar, se ha interrogado sobre las causas de esta colaboración cuestionando las explicaciones quizás demasiado simplistas que consideran que el impulso por parte de los Estados de este tipo de alianzas tiene por objetivos la búsqueda de la eficacia y la reducción de costes. En cuarto lugar, ha subrayado de forma quizás más decidida, la transformación de la restricción migratoria en una esfera de la gestión sometida a procesos de comercialización y mercantilización orientada, modulada, por la obtención de beneficios (económicos para las empresas, pero en este caso, políticos para los Estados) (Gammeltoft-Hansen, 2013; Rodier, 2012). Mientras que las necesidades del mercado de trabajo y las crecientes restricciones a la inmigración han convertido a empresarios y migrantes en «consumidores» rentables de los servicios que ofrece la industria migratoria de la facilitación, el deseo de los Estados de cumplir sus objetivos de restricción, o de cierre migratorio, les ha llevado a demandar una oferta de servicios dirigida a lograr un alto grado de eficacia -y eficiencia- en el control del acceso y el establecimiento.

En una aportación pionera en esta sección de la investigación sobre la industria migratoria, Menz subrayó a finales de la década pasada que la incorporación de actores privados a la gestión del control migratorio respondía a la deriva neoliberal del Estado en muchos países occidentales (Menz, 2009). En publicaciones 
más recientes ha ido matizando esta hipótesis, que cobra fuerza a través de un estudio comparado entre distintos casos en Europa y Norteamérica, concluyendo que la intensidad de la «privatización» del control migratorio variaba de acuerdo con el grado de neoliberalización del Estado (Menz, 2011, 2013) ${ }^{12}$.

Más recientemente la investigación sobre la industria migratoria en el campo de la restricción de la movilidad han tenido un alcance menos genérico y más específico. En esta órbita encontramos tres áreas de investigación fundamentales, y en expansión, según esferas del control y tipo de actores. Por un lado, la que se detiene en el filtrado prefronterizo y la regulación documental del acceso (Sánchez-Barrueco, 2017). Por otro, la que se ha centrado su atención en la infraestructura fronteriza y la vigilancia de los contornos terrestres, aéreos y marítimos (Lemberg-Pedersen, 2013; Baird, 2016). Y, por último, la que se interesa en las infraestructuras de detención e internamiento de inmigrantes (Flynn, 2017) ${ }^{13}$.

Las aportaciones realizadas por la literatura sobre la industria de la restricción han dialogado en menor medida con la teoría migratoria, pero su influencia ha sido destacable en los estudios sobre la criminalización de la movilidad, los derechos humanos y la economía política de las migraciones. En concreto, la delegación de parte de las funciones del control de la movilidad transfronteriza a actores privados, principalmente empresas, ha generado un intenso debate académico en torno a diversas preocupaciones que discurren por sus efectos sobre la soberanía y la jurisdicción de los Estados, su legitimidad y su supervisión pública. El análisis de sus efectos se ha centrado, por un lado, en torno al poder estructurante de las empresas de seguridad en esta área de gestión y su creciente capacidad para ejercer influencia en la toma de decisiones (Golash-Boza, 2009; Lemberg-Pedersen, 2013). Algunos autores han mostrado su preocupación, sus reticencias ante los denominados «efectos de captura» (lock-in effects) que provocan una auto-perpetuación de este tipo de prácticas más allá de coyunturas políticas y económicas (Menz, 2011). Otros análisis han subrayado los efectos de esta privatización en el progresivo desplazamiento del conocimiento experto fuera del ámbito estatal, lo que implica que los Estados reformulen sus estrategias y técnicas de control en base a una especialización y conocimiento que necesariamente debe ser provisto por actores externos (Bloom, 2015); lo que, en última instancia, puede llegar a suponer formas de dependencia y amplias limitaciones para implantar este tipo de acciones al margen de la colaboración del sector privado (López-Sala y Godenau, 2017).

En este sustrato ha surgido también una interesante discusión en torno a si estas prácticas suponen una menor implicación del Estado en la regulación migratoria. Así mientras que inicialmente se sostuvo que estos procesos suponían

${ }^{12}$ En concreto, Menz (2010) introduce la hipótesis de que pueden observarse «distintas variedades del neoliberalismo», apreciándose un mayor grado de participación de compañías privadas en las funciones de control migratorio en aquellos casos nacionales en los que este modelo es más pronunciado.

${ }^{13}$ Estas áreas han indagado en la intervención de empresas tecnológicas, de gestión de visados y de seguridad que construyen y mantienen infraestructuras fronterizas, gestionan centros de internamiento, regulan el acceso territorial y suministran equipamiento para la vigilancia. 
una «retirada» o una «retracción» del Estado» (Guiraudon y Lahav, 2000), más recientemente ha primado la tesis contraria ${ }^{14}$. Objeto de análisis ha sido también efecto de esta «privatización» en la protección efectiva de los derechos de los inmigrantes irregulares en muchos de los escenarios y dispositivos donde se ejerce el control fronterizo e interno. Estas prácticas de delegación del control migratorio, concluyen diversos autores, difuminan la responsabilidad legal de los Estados (Gammeltoft-Hansen, 2011, 2016; Humphris y Sigona, 2019), propician la falta de transparencia y alejan las acciones del Estado del escrutinio público y de la supervisión institucional (Bacon, 2005; Fernandes, 2007).

\section{LOS «OTROS» EN LA INDUSTRIA MIGRATORIA}

La esfera que ha tenido por el momento un menor recorrido en la clasificación compilatoria de Nyberg-Sorensen y Gammeltoft-Hansen (2012) es probablemente la referida a la denominada industria del rescate o industria de la asistencia $^{15}$ (Gammeltoft-Hansen y Nyberg-Sorensen 2013). Más allá del éxito o de la acogida académica de esta etiqueta, podemos destacar que su acierto ha sido abrir una discusión de gran alcance, en la literatura de intersección entre la industria migratoria y los procesos de privatización de la gestión migratoria, en torno a la función de otros actores no públicos que intervienen en la movilidad internacional: las organizaciones no gubernamentales y humanitarias y las organizaciones de migrantes.

$\mathrm{Su}$ incorporación analítica ha generado ciertos avances en este enfoque. En primer lugar, esta literatura se ha interrogado sobre la obtención de beneficios como eje motriz y articulador de esta industria. Este aspecto que rodea e inscribe las motivaciones de la acción en el caso de otros actores privados pierde fuerza en el caso de este tipo de organizaciones, que actúan llevadas por razones humanitarias, desviándose su encaje explicativo, por tanto, del modelo genérico ${ }^{16}$.

En segundo lugar, ha puesto en cuestión una atribución directa y mecánica entre actores y funciones trascendiendo la dicotomía rígida entre las funciones de

14 Menz, por ejemplo, ha sostenido de forma contundente que resulta erróneo afirmar que los Estados han perdido su capacidad de influencia en el diseño y aplicación de las políticas de control migratorio. Lo observado responde, en su opinión, al desarrollo de nuevas fórmulas de gestión que implican nuevas tácticas, nuevos canales y nuevos mecanismos que evidencian la expansión del poder punitivo y represivo del Estado (Menz, 2013).

${ }_{15}$ Se trata de un término prestado de la obra de 2007, Sex at the Margins (véase Agustín, 2007). En opinión de estos autores las organizaciones no gubernamentales y humanitarias han llegado a convertirse en una significativa subcategoría dentro de la industria migratoria (GammeltoftHansen y Nyberg-Sorensen, 2013, 10).

16 La centralidad de la obtención de beneficios ha sido también cuestionada en algunos estudios etnográficos recientes sobre el papel de los facilitadores de la inmigración irregular. Estos han ofrecido, a través de sus conclusiones, una contranarrativa que se desvía de la visión criminalizadora de los «traficantes» como explotadores de migrantes y en donde destacan otros principios, como el sentido de comunidad, identidad, reciprocidad, apoyo mutuo y confianza (véase, por ejemplo, Stone-Cadena y Álvarez, 2018). 
facilitación y restricción ${ }^{17}$. El análisis de las organizaciones humanitarias desde esta perspectiva ha permitido destacar la diversidad de sus tareas y la versatilidad de sus roles. La participación de organizaciones sociales en la movilidad internacional es habitualmente resultado de su intento de suplir la falta de instrumentos institucionales para proteger los derechos de los migrantes irregulares durante sus trayectos migratorios lo que supone generalmente entrar en conflicto con los objetivos y las lógicas de gestión migratoria del Estado (Nyberg-Sorensen, 2013; Ambrosini, 2017). Sin embargo, los estudios sobre la formas que ha adoptado la delegación del control migratorio ha evidenciado también su cooperación con los gobiernos en muchos de los dispositivos de control migratorio a través de la firma de convenios de colaboración, que incluyen, entre otros, la participación en programas de retorno, la provisión de servicios en centros de internamiento o la gestión de centros de menores inmigrantes y de acogida de solicitantes de asilo y refugio. Por ello, los estudios realizados en esta área han destacado los potenciales efectos de este tipo de acuerdos en la autonomía, la independencia y la capacidad de contestación de las organizaciones sociales. Los análisis se han detenido en los peligros de la cooptación y en la creciente dependencia económica de una parte de estas organizaciones. En opinión de algunos autores, esta dependencia no sólo termina por moldear significativamente su agenda, sino que impulsa una «cultura institucional de la subvención» (Tyler, Gill, Conlon y Oeppen, 2014) que debilita la contestación y su incidencia política. De ahí, señalan, que muchas organizaciones sociales hayan terminado por desarrollar una agenda de intervención «acrítica» con las políticas de control, por acciones meramente instrumentales, centradas en el suministro de servicios, sometidas a una amplia supervisión y bajo el escrutinio de las administraciones públicas (Humphris y Sigona, 2019).

\section{CONCLUSIONES}

A pesar de que el enfoque de la industria migratoria necesita de mayor recorrido teórico, en diálogo con otras perspectivas, y de cierto empaque empírico, a través de la incorporación de nuevos actores y estudios de caso, sus aportaciones han abierto un campo de reflexión muy fructífero para comprender con más profundidad los procesos migratorios contemporáneos. A pesar de su fragmentación, de su parcelación a lo largo de distintas esferas, casos y actores, sus contribuciones han servido fundamentalmente para profundizar en la complejidad y en la multiplicidad de formas que ha adoptado la articulación entre los migrantes, el sector privado, los Estados y las organizaciones sociales en los procesos migratorios. El examen de las industrias migratorias ha colocado en el centro del análisis la mercantilización y la comercialización de la movilidad humana, impulsando el estudio y la sistematización de la diversidad de formas de intermediación que intervienen, condicionan y dan forma a los flujos. Ha tenido tam- 
bién la virtud de evidenciar la creciente influencia de los actores privados en la gobernanza global de la movilidad humana y de aportar nuevos contenidos a las orientaciones que pretenden superar el nacionalismo metodológico a través del análisis de las redes comercializadas y de los actores informales especializados en facilitar la movilidad transfronteriza en el seno de los regímenes migratorios restrictivos. También ha tenido un papel fundamental en dar profundidad explicativa y densidad analítica a los estudios sobre la delegación y la externalización de la gestión migratoria trascendiendo las aportaciones meramente descriptivas sobre la privatización regulatoria en esta área. Este enfoque se ha sumado a las perspectivas explicativas sobre la continuidad y el mantenimiento de los flujos migratorios, entrando en diálogo con la teoría de redes sociales y, más recientemente, ha completado otros modelos explicativos sobre la activación, la composición y la dirección de los flujos.

Sus aportaciones han sido especialmente destacables en la investigación sobre los flujos migratorios laborales y, más recientemente, aunque con menor recorrido, en el análisis de la restricción migratoria y de la gestión de la acogida. Esta literatura ha profundizado en los efectos de la intermediación laboral en la conformación de los patrones migratorios, la estructuración del mercado de trabajo de los países de acogida y el impacto sobre la precariedad laboral de las poblaciones migrantes. También ha logrado aportar luz en torno a los vínculos entre distintos actores en la gestión de la movilidad internacional de trabajadores. En la esfera de la restricción migratoria, la industria migratoria ha dado buena cuenta de la multiplicidad de fórmulas de colaboración existentes entre el Estado y las empresas privadas tecnológicas y de seguridad que han llegado a convertirse en parte fundamental de la implantación de esta política pública. También ha albergado un interesante debate en torno a las lógicas explicativas de este «giro» en las prácticas del control, así como sus implicaciones, entre otros, sobre la soberanía, la legitimidad, la supervisión pública de la acción estatal y la protección de los derechos de los inmigrantes irregulares. Por último, la inclusión analítica de las organizaciones sociales y de inmigrantes a través de la denominada industria de la asistencia se ha interrogado sobre los efectos de estas coaliciones Estado-sociedad civil en la erosión de la independencia y la capacidad de incidencia de estas organizaciones.

\section{BIBLIOGRAFÍA}

AGUSTIN, L (2007) Sex at the Margins. Migration, Labour Markets and the Rescue Industry, Londres, Zed Books.

AMBROSINI, M. (2017): "Why Irregular Migrants Arrive and Remain: The Role of Intermediaries", Journal of Ethnic and Migration Studies, 43, 11. pp. 1813- 1830.

ANDRIJASEVIC, R. (2015): "The Commercialisation of Migration Control”, en Citizenship and its Others, Basingstoke. Palgrave Macmillan, pp. 89-94. 
BAAS, M. (2018): "What Determines the Cost of Migration? A Perspective from Indian Agents facilitating migration to Singapore and the Middle East", Working Paper Series, 264, Singapur, Asia Research Institute.

BACON, C. (2005): "The Evolution of Immigration Detention in the UK: The Involvement of Private Prison Companies", Working Papers Series, 27 (September).

BAIRD, T. (2016): "Surveillance Design Communities in Europe: A Network Analysis", Surveillance and Society, 14, 1, pp. 34-58.

BETTS, A. (2013): "The Migration Industry in Global Migration Governance", en The Migration Industry and the Commercialization of International Migration, Londres. Routledge, pp. 45-63.

BLOOM, T. (2015): "The Business of Migration Control: Delegating Migration Control functions to Private Actors", Global Policy, 6, pp. 151-157.

BLOOM, T. (2016): “The Business of Noncitizenship”, Citizenship Studies, 19, pp. 892906.

CALAVITA, K. (1992): Inside the State: The Bracero Program, Immigration, and the INS, Nueva York, Routledge.

CASTLES, S. y MILLER, M. J. (2003): The Age of Migration. International Population Movements in the Modern World, Nueva York, Palgrave McMillan.

CASTLES, S. (2007): "The Factors That Make and Unmake Migration Policies", en Rethinking Migration: New Theoretical and Empirical Perspectives, Nueva York, Berghahn, pp. 29-61.

COHEN, R. (1997): Global Diasporas: An Introduction, Seattle, University of Washington Press.

CONSTERDINE, E. y SAMUK, S. (2018): “Temporary Migration Programmes: The Cause or Antidote for Migrant Worker Exploitation in UK Agriculture", Journal of International Migration and Integration, 19, 4, pp. 1005-1020.

CRANSTON, S.; SCHAPENDONK, J. y SPAAN, E. (2018): "New Directions in Exploring The Migration Industries", Journal of Ethnic and Migration Studies, 44, 4, pp. 543-557.

CRESSWELL, T. (2006): On the Move. Mobility in the Western World, Londres, Taylor and Francis.

FAIRCHILD, H. P. (1925): Immigration: A World Movement and its American Significance, Nueva York, Macmillan.

FERNANDES, D. (2007): Targeted: Homeland Security and the Business of Immigration, Nueva York, Seven Stories Press.

FINDLAY, A. y McCOLLUM, D. (2013): "Recruitment and Employment Regime: Migrant Labour Channels in the UK's Rural Agrobusiness Sector from Accession to Recession", Journal of Rural Studies, 30, pp. 10-19.

FLYNN, M. (2017): "Detained beyond The Sovereign. Conceptualising Non-State Actor Involvement in Immigration Detention" en Intimate Economies of Immigration Detention, Londres, Routledge, pp.15-31.

FRANCK, A. ARELLANO, E. y ANDERSON. J. (2018): "Navigating Migrant Trajectories Through Private Actors: Burmese Labour Migration to Malaysia", European Journal of East Asian Studies, 17, 1, pp. 55-82.

GABACCIA, D. (2000): Italy's Many Diasporas, Londres, UCL Press.

GALARZA, E. (1964): Merchants of Labor: The Mexican Bracero Story, Santa Bárbara, McNally and Loftin. 
GAMMELTOFT-HANSEN, T. (2011): Access to Asylum: International Refugee Law and The Globalisation of Migration Control, Cambridge, Cambridge University Press.

GAMMELTOFT-HANSEN, T. y NYBERG-SORENSEN, N. (eds.) (2013): The Migration Industry and the Commercialization of International Migration, Londres. Routledge.

GAMMELTOFT-HANSEN, T. (2016): "Private Security and the Migration Control Industry" en Routledge Handbook of Private Security Studies, Londres, Routledge, pp. 207-213.

GARAPICH, P. (2008): “The Migration Industry and Civil Society. Polish Immigrants in the United Kingdome before and after EU Enlargement", Journal of Ethnic and Migration Studies, 34, 5, pp. 735-752.

GOH, C; WEE, C. y YEOH, B. (2017): "Migration Governance and The Migration Industry in Asia: Moving Domestic Workers from Indonesia to Singapore", International Relations of the Asia-Pacific, 17, pp. 401-433.

GOLASH-BOZA, T. (2009): "The Immigration Industrial Complex: Why We Enforce Immigration Policies Destined to Fail”, Sociology Compass, 3, 2, pp. 295-309.

GROUTSIS, D; VAN DEN BROEK, D. y HARVEY, W. (2015): Transformations in Network Governance: the Case of Migration Intermediaries", Journal of Ethnic and Migration Studies, 41, 10, pp. 1558-1577.

GUIRAUDON, V. y LAHAV, G. (2000): "A Reappraisal of the State Sovereignty Debate. The Case of Migration Control”, Comparative Political Studies, 33, 2, pp. 163 195.

HARNEY, F. (1977): “The Commerce of Migration”, Canadian Ethnic Studies, 9, 1, pp. 42-53.

HENNEBRY, J. (2008): "Bienvenidos a Canadá. Globalization and the Migration Industry Surrounding Temporary Agricultural Migration in Canada", Canadian Studies in Population, 35, 2, pp. 339-356.

HERNÁNDEZ-LEÓN, R. (2005): The Migration Industry in the Mexico-U.S. Migratory System, Los Angeles, California Center for Population Research.

HERNÁNDEZ-LEÓN, R. (2012): "La Industria de la Migración en el Sistema Migratorio México-Estados Unidos", Trace, 61, pp. 41-61.

HERNÁNDEZ-LEÓN, R. (2013). "Conceptualizing the Migration Industry” en The Migration Industry and the Commercialization of International Migration, Nueva York, Routledge, pp. 24-43.

HERNÁNDEZ-LEÓN, R. y SANDOVAL, E. (2017): “El Reclutamiento de Trabajadores Temporales Mexicanos para Estados Unidos: Infraestructura Burocrática, Industria de la Migración y Economía del Engaño en el Programa de Visas H-2", UCLA International, pp. 1-33.

HUMPHRIS, R. y SIGONA, N. (2019): “Outsourcing the Best Interest of Unaccompanied Asylum Seeking Children in The Era of Austerity", Journal of Ethnic and Migration Studies, 45, 2, pp. 312-330.

JENS, J. W. y LAUCK, W. J. (1922): The Immigration Problem, Nueva York, Funk and Wagnalls.

KEMP, A. y RAIJMAN, R. (2014): "Bringing in State Regulations, Private Brokers, and Local Employers: a Meso-level Analysis of Labor Trafficking in Israel", International Migration Review, 48, 3, pp. 604-642. 
KERN, A. y MÜLLER-BÖKER, U. (2015): “The Middle Space of Migration: A Case Study on Brokerage and Recruitment Agencies in Nepal”, Geoforum, 65, pp. 158169.

FERNÁNDEZ-MONTES, M. (2013) (coord.): Negociaciones identitarias en contextos migratorios, Madrid, Common Ground Publishing.

KRISSMAN, F. (2000): Immigrant Labor Recruitment: U.S Agribusiness and Undocumented Migration from Mexico", en Immigration Research for a New Century, Nueva York, Russell Sage Foundation, pp. 277-301.

KRISSMAN, F.(2005): "Sin Coyote Ni Patrón: Why The Migrant Network Fails to explain International Migration", International Migration Review, 39, 1, pp. 4-44.

KUSHNIROVICH, N; RAIJMAN, R. y BARAK-BIANCO, A. (2018): "The Impact of Government Regulation on Recruitment Process, Rights Wages and Working Conditions of Labor Migrants in the Israeli Construction Sector", European Management Review, DOI: 10.1111/emre.12169.

KYLE, D. y KOSLOWSKI, R. (2001): Global Human Smuggling: Comparative Perspectives, Washington, John Hopkins University.

LAHAV, G. (1998): "Immigration and the State: The Devolution and Privatisation of Immigration Control in the EU", Journal of Ethnic and Migration Studies, 24, 4, pp. 675-694.

LAHAV, G. (2000): "The Rise of Nonstate Actors in Migration Regulation in the United States and Europe: Changing the Gatekeepers or Bringing back the State" en Immigration Research for a New Century: Multidisciplinary Perspectives, Nueva York, Russell-Sage Foundation, pp. 215-241.

LEMBERG-PEDERSEN, M. (2013): "Private Security Companies and the European Borderscapes", en The Immigration Industry and the Commercialization of International Migation, Londres, Routledge, pp. 152-172.

LINDQUIST, J.; XIANG, B. y YEOH, B. (2012): “Opening the Black Box of Migration: Brokers, the Organization of Transnational Mobility and the Changing Political Economy in Asia", Pacific Affairs, 85, 1. pp. 7-19.

LIGHT, I. (2013): “The Migration Industry in the United States, 1882-1924.”, Migration Studies, 1, 3. pp. 258-275.

LÓPEZ-SALA, A. y GODENAU, D. (2016): "Non-State Actors and Migration Control in Spain". Third ISA Forum of Sociology. Viena, Austria, disponible en http://digital.csic.es/bitstream/10261/136847/1/Paper\%20Viena.pdf.

LÓPEZ-SALA, A. y GODENAU, D. (2017): "Delegando el control migratorio. Una aproximación a las prácticas del Outsourcing en España”, en Estados de contención, Estados de detención. El control de la inmigración irregular en España, Barcelona, Anthropos, pp. 197-223.

LÓPEZ-SALA, A. y GODENAU, D. (2019): "Non-state Actors and the Outsourcing of Migration Control in Spain" en Migrants and Refugees in Times of Crisis, Atenas, EPLO.

MARTIN, P. (2005): Merchants of Labor. Agents of the Evolving Migration Infrastructure, Ginebra, International Institute for Labour Studies.

MASSEY, D; ALARCÓN, R; DURAND, J. y GONZÁLEZ, H. (1987): Return to Aztlan. The Social Process of International Migration from Western Mexico, Berkeley, University of California Press.

McKEOWN, A. (2012): "How the Box Became Black: Brokers and the Creation of the Free Migrant”, Pacific Affairs, 85, 1, pp. 21-45. 
MENZ, G. (2009): “The Neoliberalized State and Migration Control: The Rise of Private Actors in the Enforcement and Design of Migration Policy", Journal of Contemporary Central and Eastern Europe, 17, 3, pp. 315-332.

MENZ, G. (2011): "Neoliberalism, Privatisation and the Outsourcing of Migration Management: A Five Country Comparison", Competition and Change, 15, 2, pp. 116135.

MENZ, G. (2013): "The Neoliberalized State and the Growth of the Migration Industry" en The Migration Industry and the Commercialization of International Migration, Londres, Routledge, pp. 108-127.

NYBERG-SORENSEN, N. (2012): "Revisiting the Migration-Development Nexus: from Social Networks and Remittances to Markets for Migration Control", International Migration, 50, 3, pp. 61- 76.

NYBERG-SORENSEN, N. (2013): "Migration between Social and Criminal Networks: Jumping the remains of the Honduran Migration Train" en The Migration Industry and the Commercialization of International Migration, Londres, Routledge, pp. 238261.

NYBERG-SORENSEN, N. y GAMMELTOFT-HANSEN, T. (2012): The Migration Industry and Future Directions for Migration Policy, Copenhage, Danish Institute for International Studies.

PEDREÑO, A. (2014): De cadenas, migrantes y jornaleros. Los territorios rurales en las cadenas globales agroalimentarias, Madrid, Talasa.

RODIER, C. (2012): El negocio de la xenofobia, Madrid, Clave Intelectual.

SALT, J. y STEIN, J. (1997): "Migration as Business. The Case of Trafficking", International Migration, 35, pp. 467-493.

SÁNCHEZ-BARRUECO, L. (2017): "Business as Usual? Mapping Outsourcing Practices in Schengen Visa Processing" Journal of Ethnic and Migration Studies, 44, 3, pp. 384-400.

SCHAPENDONK, J. y STEEL, G. (2014): "Following Migrant Trajectories. The In/ mobility of Sub-Saharan African en route to the European Union", Annals of the Association of American Geographers, 104, 2, pp. 262-270.

SCHAPENDONK, J. (2018):"Navigating the Migration Industry: Migrants moving through an African-European Web of Facilitation/Control", Journal of Ethnic and Migration Studies, 44, 4, pp. 663-679.

SHELLER, M. y URRY, J. (2006): “The New Mobilities Paradigm”, Environment and Planning, 38, pp. 207-236.

SPENER, D. (2009): Clandestine Crossings. Migrants and Coyotes on the Texas-Mexico Border, Ithaca, Cornell University Press.

SPENER, D. (2010): Some Critical Reflections on the Migration Industry Concept, disponible en http://faculty.trinity.edu/dspener/clandestinecrossings/related\%20articles/ migration\%20industry.pdf.

SPENER, D. (2011): "Global Apartheid, Coyotaje, and The Discourse of Clandestine Migration" en Global Smuggling. Comparative Perspectives. Baltimore, John Hopkins University Press, pp. 157-185.

SPAAN, E. y VAN NAERSSEN, T. (2018): "Migration Decision-making and Migration Industry in the Indonesia-Malaysia Corridor", Journal of Ethnic and Migration Studies, 44, 4, pp. 680-695.

STONE-CADENA, V. y ÁLVAREZ, S. (2018): "Historicizing Mobility: Coyoterismo in the Indigenous Ecuadorian Migration Industry", Annals of the American Academy of Political and Social Science, 676, pp. 194-211. 
SURAK, K. (2018): "Migration Industries and the State: Guestwork Programs in East Asia", International Migration Review, 52, 2, pp. 487-523.

TYLER, I; GILL, N. CONLON, D. y OEPPEN, C. (2014): "The Business of Child Detention: Charitable Co-option, Migrant Advocay and Activist Outrage", Race and Class, 56, 1, pp. 3-21.

VAN DEN BROEK, D; HARVEY, W. y GROUTSIS, D. (2016): “Commercial Migration Intermediaries and the Segmentation of Skilled Migrant Employment", Work, Employment and Society, 30,3, pp.523-534.

XIANG, B. (2012): "Predatory Princes and Princely Peddlers: the State and International Migration Intermediaries in China", Pacific Affairs, 85, 1. pp. 47-68.

XIANG, B. y LINDQUIST, J. (2014): «Migration Infrastructure», International Migration Review, 48, 1. pp. 122-148.

ZABKO, O.; AASLAND, A. y ENDRESEN, S. B. (2018): "Facilitating Labour Migration from Latvia: Strategies of Various Categories of Intermediaries", Journal of Ethnic and Migration Studies, 44,4, pp. 575-591. 